\title{
Experimental and theoretical observations on DDT in smooth narrow channels
}

\author{
J. Melguizo-Gavilanes ${ }^{\mathrm{a}, 1, *}$, Y. Ballossier ${ }^{\mathrm{a}}$, L. M. Faria ${ }^{\mathrm{b}}$ \\ ${ }^{a}$ Institut Pprime, UPR 3346 CNRS, ISAE-ENSMA, 86961 Futuroscope-Chasseneuil \\ Cedex, FRA \\ ${ }^{b}$ ENSTA ParisTech, CNRS, INRIA, Université Paris-Saclay, 91762 Palaiseau Cedex, \\ FRA
}

\begin{abstract}
A combined experimental and theoretical study of deflagration-to-detonation transition (DDT) in smooth narrow channels is presented. Some of the distinguishing features characterizing the late stages of DDT are shown to be qualitatively captured by a simple one-dimensional scalar equation. Inspection of the structure and stability of the traveling wave solutions found in the model, and comparison with experimental observations, suggest a possible mechanism responsible for front acceleration and transition to detonation. Keywords: flame acceleration, DDT experiments, narrow channels, simple model, non-ideal detonations
\end{abstract}

\footnotetext{
${ }^{*}$ Corresponding author

Email address: josue.melguizo-gavilanes@cnrs.pprime.fr (J. Melguizo-Gavilanes) 


\section{Introduction}

Reactive mixtures in confined geometries may detonate if ignited. Small flames or sparks can accelerate and undergo deflagration-to-detonation transition (DDT), which, in most cases, presents significant safety hazards [1, 2], and, in others, can also be used to produce thrust or to generate power [3]. Understanding of the fundamental mechanisms of DDT continues to be a challenge in combustion theory. The specific details of DDT are dependent on many factors including channel geometry and size. Nevertheless, DDT, in general, proceeds through a series of distinct stages [2, 4]. Ignition of a deflagration drives a flow in the reactants and produces compression waves that propagate in front of the flame. This flow and the resulting fluctuations stretches the flame and increases its surface area, which, in turn, accelerates the flame further. Eventually, compression waves generated by the accelerating flame coalesce to produce a precursor shock. Feedback between the accelerating flame and shock compression subsequently ignites the gas and initiate a detonation.

Different mechanisms of DDT have been observed in numerical simulations of smooth channels. One mechanism involves viscous heating of the boundary layers in the flow induced by the precursor shock that forms ahead of the flame. The shock and viscous dissipation can heat the reactive material in the boundary layer to a condition where autoignition can occur in the time between the passage of the shock and the arrival of the flame [58]. Another mechanism involves localized pressure increases near the flame, which enhances the local burning rate, which, in turn, leads to further local pressure increases. This feedback can produce a shock, which compresses the 
unburned material in front of the flame and induces conditions that lead to a detonation [9].

In this manuscript, we link experimental observations of the dynamics of DDT with a simple theoretical model with generic losses (i.e. curvature and friction) recently described in the context of detonation dynamics [10]. In the case of friction losses, the model admits two steady traveling wave solutions: (i) typical structure of a detonation in which chemical reaction is activated immediately behind the leading shock wave; (ii) a reaction zone that trails far behind the leading shock wave. Notably, state (ii), generally unstable, always transitions into state (i) if perturbed. The nature of this transition bears striking similarities with what is typically observed during DDT experiments in narrow channels.

\section{Experimental observations of DDT in narrow channels}

\subsection{Experimental Setup}

The experimental campaign was performed at the Karlsruhe Institute of Technology (KIT) in Germany during a research visit in the summer of 2011. Two different channels were used, $6 \mathrm{~mm} \times 6 \mathrm{~mm}$ and $10 \mathrm{~mm} \times 10$ $\mathrm{mm}$, both $1 \mathrm{~m}$ in length. Over 100 experiments for $\mathrm{H}_{2}-\mathrm{O}_{2}$ mixtures with $\mathrm{H}_{2}$ concentrations by volume varying from 30 to $70 \%$ were carried out. The goal of the study was to visualize the different propagation regimes as a function of $\mathrm{H}_{2}$ concentration, determine run-up distances, build $x-t$ diagrams and attempt to capture the DDT event in as much detail as possible. The channels were made by cutting a $1 \mathrm{~m}$ in length by the channel height (6 or

$10 \mathrm{~mm}$ ) from a solid aluminum sheet of length $1.20 \mathrm{~m}$, height $0.25 \mathrm{~m}$, and 
thickness $6 / 10 \mathrm{~mm}$. Three of the five walls needed for a square cross-section close end-open end channel resulted from this cutting procedure (left, top and bottom walls). The remaining two faces, front and back walls, were made of plexiglass sheets $(1.2 \mathrm{~m} \times 0.25 \mathrm{~m} \times 10 \mathrm{~mm})$ to allow for optical access to capture the entire DDT evolution. The plexiglass sheets were glued to the aluminum sheet, and held in place with steel square profiles secured with bolts to provide structural integrity (see Fig 1).
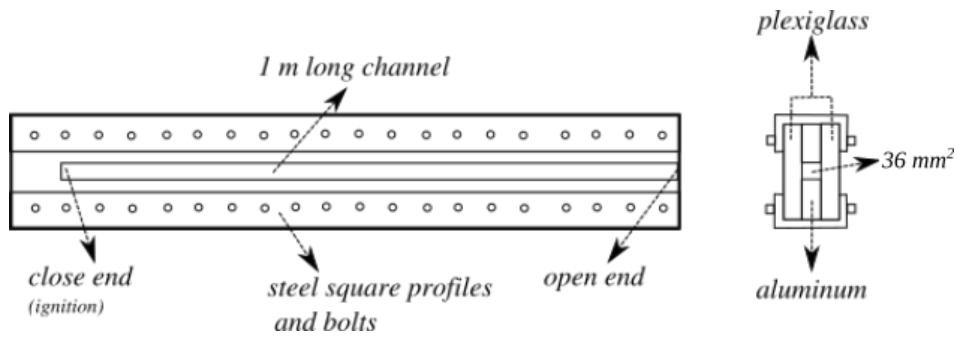

Figure 1: Schematic of experimental setup showing front and side view of the $6 \mathrm{~mm} \times 6$ mm channel (not to scale).

Finally, two metal rods with sharp points protruding from the top/bottom walls were added adjacent to the left wall (closed end of the channel) to create a weak spark to ignite the mixture using a capacitor. The separation distance between the rods and spark energy were approximately $3 \mathrm{~mm}$ and 10 mJ, respectively. The mixtures were prepared in an external vessel using the method of partial pressures and left to settle for 5 minutes before being fed to the channel from its open end. Three types of optical visualization techniques were used: direct observation (DO), shadowgraph (SG) and schlieren (SC). High speed videos of the entire DDT process were acquired using a Photron FASTCAM SA1.1 model 675K-C1. In the sections below, we focus only on the description of the DDT dynamics for stoichiometric $\mathrm{H}_{2}-\mathrm{O}_{2}$ mixtures using 
the using the $6 \mathrm{~mm} \times 6 \mathrm{~mm}$ channel.

\subsection{DDT dynamics}

Figure 2 shows an $x-t$ diagram from ignition, flame propagation, acceleration and transition to detonation obtained using SG with a $5 \mu$ s interval between frames. Note that contrary to standard convention we take $t=0$ to be at the top of the figure. The original color images were processed to sharpen gradients and color-desaturated to achieve the greyscale shown; only the first $0.3 \mathrm{~m}$ of the channel are displayed in the figure.

Typical features of this phenomenon stand out: a leading shock that forms from the steepening of the pressure wave created by the ignition event and supported in part by the propagation of the flame. An initial stage of flame propagation exhibiting a very smooth flame surface and a quasiconstant propagation speed of $337 \mathrm{~m} / \mathrm{s}$. Appearance of flame instabilities and associated increase in flame surface area visible in subsequent frames. Further acceleration is experienced by the flame accompanied by new pressure pulses/waves that slowly steepen to form a strong/thick precursor shock very close to its tip. Shortly after, the flame-precursor shock complex abruptly transitions into a detonation signaled by the bright white spot present at the bottom right corner of the figure. The run-up distance and flame speed prior to DDT were $0.262 \mathrm{~m}$ and $1,430 \mathrm{~m} / \mathrm{s}$, respectively. The experimentally observed detonation velocity right after DDT was $2,783 \mathrm{~m} / \mathrm{s}$.

Figure 3 summarizes all the stages observed. Note that to capture the extra frames shown on the right of this figure $-D D T$ and detonation propagation-, an additional experiment was run with the same configuration but with a modified field of view $(0.2-0.5 \mathrm{~m})$. This was done to capture the 


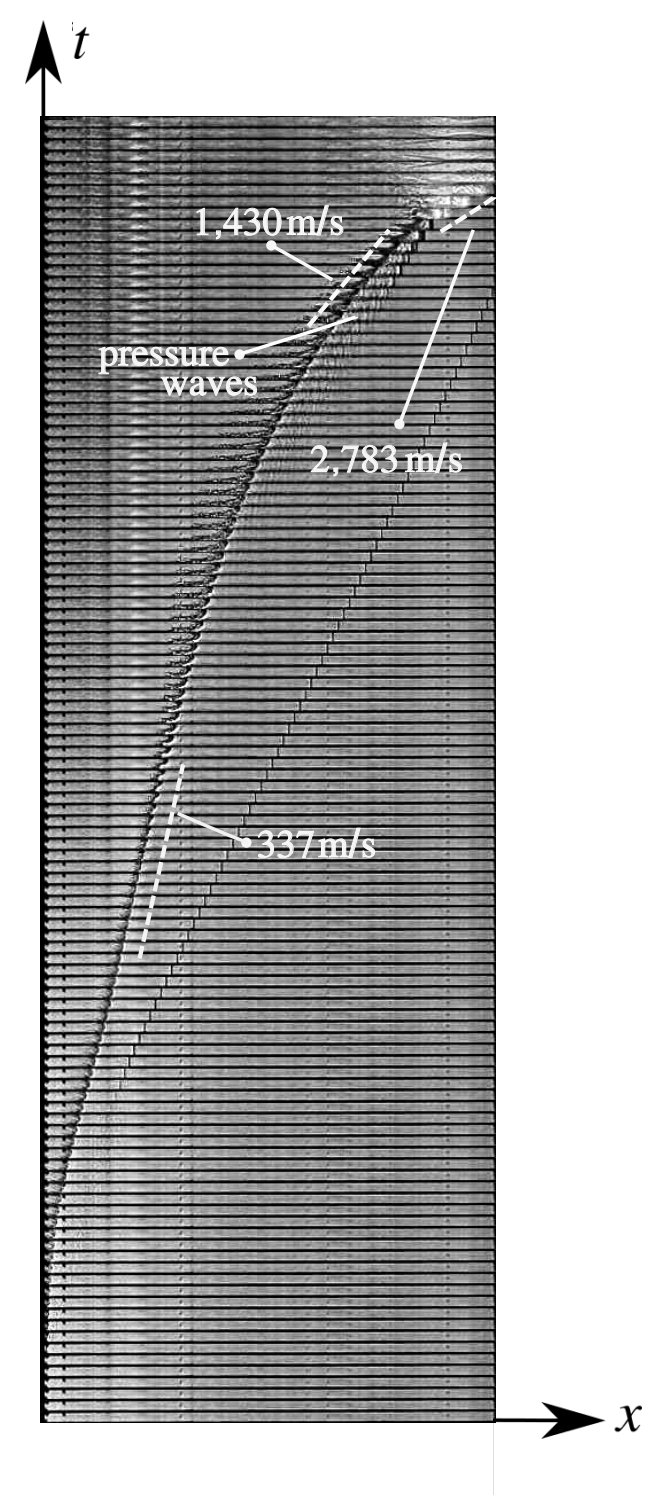

Figure 2: Experimental $x-t$ diagram for stoichiometric $\mathrm{H}_{2}-\mathrm{O}_{2}$ in a $6 \times 6 \mathrm{~mm}$ channel. Initial conditions: $\mathrm{p}_{0}=100 \mathrm{kPa}, \mathrm{T}_{0}=295 \mathrm{~K}$. Horizontal axis: length of channel from 0 $-0.3 \mathrm{~m}$; Vertical axis: time showing 114 frames with a time interval $\Delta t=5 \mu \mathrm{s}$.

DDT event and detonation propagation in more detail. The temporal resolution was increased capturing frames at $2.5 \mu \mathrm{s}$. The steepening of pressure 
waves generated by the accelerating flame were better resolved from the first frame shown. The flame rapidly catches up with the leading shock and transition to detonation takes place. The run-up distance and flame speed before DDT were $0.267 \mathrm{~m}$ and $1,484 \mathrm{~m} / \mathrm{s}$, respectively, attesting for the repeatability of the experiment. The steady detonation velocity recorded during late stages of propagation was measured to be $2,518 \mathrm{~m} / \mathrm{s}$, about $11 \%$ less than the detonation velocity predicted from ideal Chapman-Jouguet theory for this mixture $(2,837 \mathrm{~m} / \mathrm{s})$. This deficit is a characteristic feature of detonation propagation in narrow channels where friction and heat losses play a role in its dynamics [11].
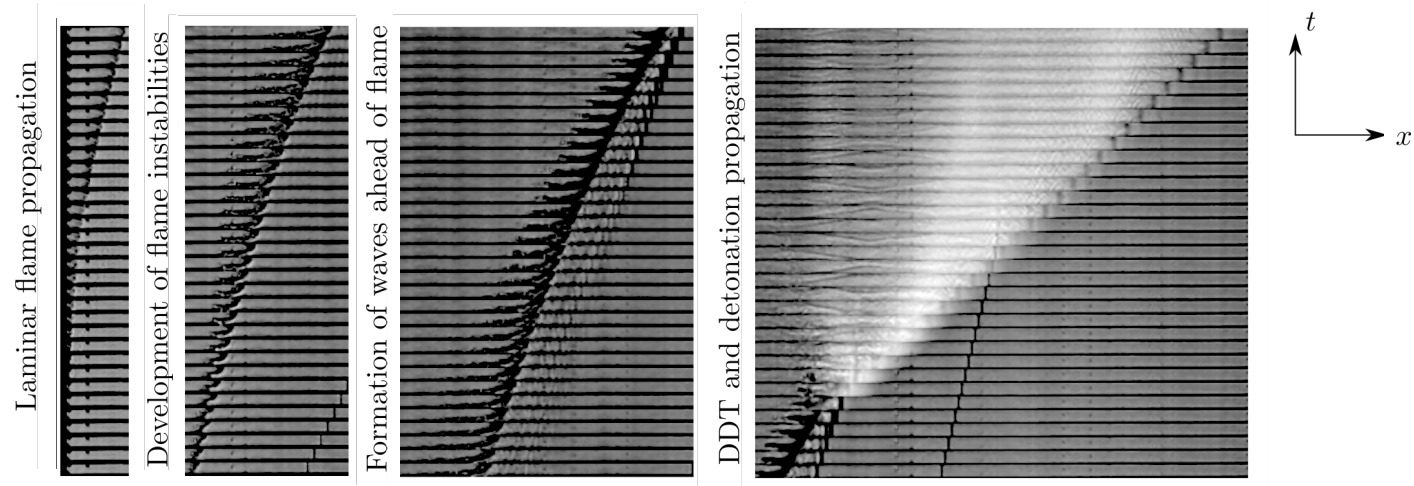

Figure 3: DDT stages for stoichiometric $\mathrm{H}_{2}-\mathrm{O}_{2}$ in a $6 \times 6 \mathrm{~mm}$ channel. Initial conditions: $\mathrm{p}_{0}=100 \mathrm{kPa}, \mathrm{T}_{0}=295 \mathrm{~K}$. Time interval between frames: $\Delta t=5 \mu \mathrm{s}$ for laminar flame propagation, development of flame instabilities and formation of waves ahead of flame; $\Delta t$ $=2.5 \mu \mathrm{s}$ for $\mathrm{DDT}$ and detonation propagation.

Two more experiments were performed that attempted to resolve the DDT event with a reduced field of view of $0.1 \mathrm{~m}(0.25-0.35 \mathrm{~m})$ and a frame rate of $\sim 15 \mu \mathrm{s}$. See Fig. 4. These were done using a shorter exposure in order to avoid the bright spots present in the previous experiments. The transition was successfully captured in both cases. Frames $(a)$ of Fig. 4 -top 
and bottom, show the structure of the flame-precursor shock complex prior to transition consisting of a region of compressed gas between the flame and the precursor shock. While in frame $(b)$ of Fig. 4 -top the mixture seemed to have autoignited abruptly after $\sim 15 \mu \mathrm{s}$, Fig. 4-bottom shows a more gradual evolution with the flame catching up to the precursor shock between frames $(a)$ and $(b)$, finally overtaking it $\sim 15 \mu$ s later in frame $(c)$. Lastly, the transverse waves characteristic of a fully developed detonation are clearly visible, together with the retonation propagating in the opposite direction, in the last frames of the figure.

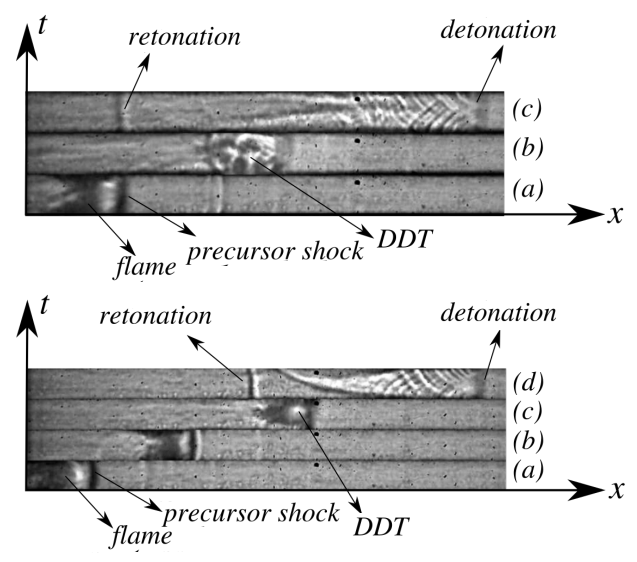

Figure 4: Close ups to DDT for stoichiometric $\mathrm{H}_{2}-\mathrm{O}_{2}$ in a $6 \times 6 \mathrm{~mm}$ channel from two different experiments using the same initial conditions $\left(\mathrm{p}_{0}=100 \mathrm{kPa}, \mathrm{T}_{0}=295 \mathrm{~K}\right)$. Horizontal axis: length of channel from $0.25-0.35 \mathrm{~m}$; Vertical axis: time with an interval $\Delta t \sim 15 \mu \mathrm{s}$. Top: autoignition between flame and precursor shock. Bottom: more gradual transition with the flame catching up with the precursor shock.

The video frames in Fig. 2, up to DDT, were postprocessed using a front tracking algorithm to extract the $x-t$ diagram shown in Fig. 5 (left). The experimental data was fitted to a global fourth order polynomial, analytically differentiated to compute the instantaneous flame velocity $\left(u_{f}\right)$ along the channel, and normalized using the sound speed in fresh mixture $\left(c_{o}=539.48\right.$ 
m/s) - see Fig. 5 (right). The deviation between the experimental data and the polynomial fit is also shown in Fig. 5 (left) for reference. The fit is poor at very early stages $(0 \leq t \leq 120 \mu \mathrm{s})$ but quickly improves thereafter with an overall deviation of less than $1 \%$ (see inset).

Next, we present a mathematical model with generic losses that is expected to be valid to describe the dynamics of the reacting front for $x / L>$ 0.1125 , location where the formation of pressure waves ahead of the accelerating flame is observed in Figs. 2 and 3.
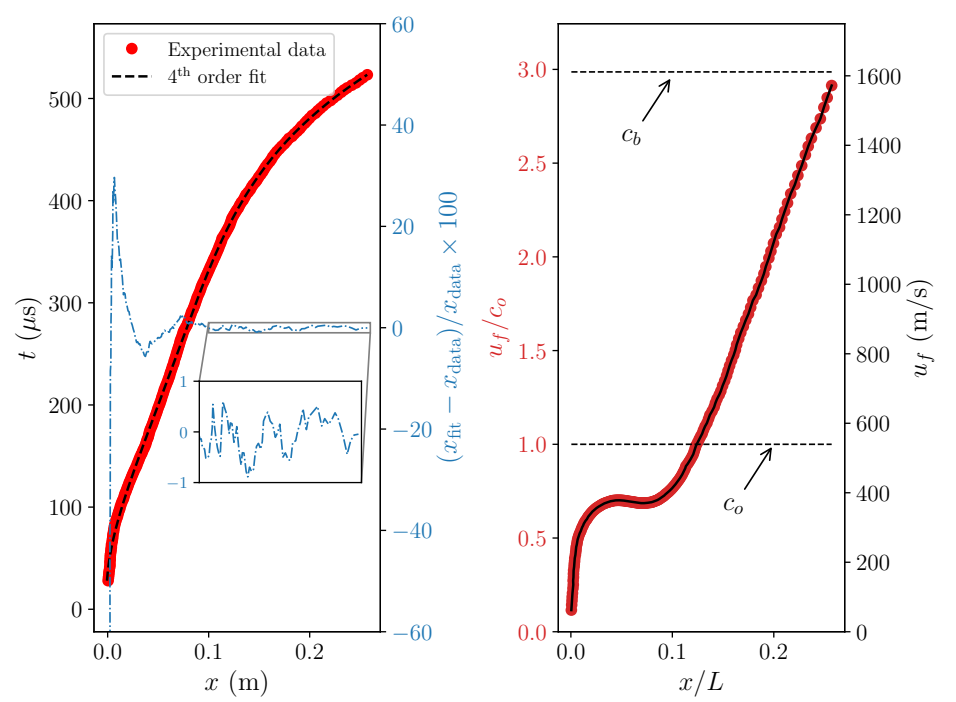

Figure 5: Left: flame front position, $x_{f}$, as a function of time, $t$, up to DDT, and deviation of fit from experimental data (dashed-dotted line). Right: normalized flame front velocity, $u_{f} / c_{o}$, as a function of normalized channel length, $x / L ; c_{o}$ and $c_{b}$ shows the speed of sound in fresh and burnt mixture. Note that $x$ and $x / L$ are equivalent because the channel length in the experiment is $L=1 \mathrm{~m}$.

\section{A model with generic losses}

Using physical arguments, Zel'dovich argued in [11] that detonation waves in narrow tubes may be mathematically described through an effectively one- 
dimensional model. Zel'dovich suggested that the tube walls act as a sink of energy and/or momentum, which may be taken into account by the inclusion of empirical source terms in the inviscid reactive Euler equations (see system of equations (1) in [11]). These models are only expected to be valid provided that the wave is sufficiently fast so that transport effects become relevant only inside boundary layers, where one may hope to replace a more complex multidimensional Navier-Stokes description by a one-dimensional Euler model with losses. A further simplification is possible by assuming that the wave is also weakly nonlinear. The latter assumption reduces the Euler system in the presence of losses to much simpler Burgers' like equations (see [12, chapter 6]). In [10], the authors proposed a simple toy model in order to study, from a qualitative point of view, certain features of detonations in the presence of curvature and friction losses.

The scalar model used ${ }^{1}$, in the laboratory frame of reference, reads:

$$
u_{t}+\left(\frac{u^{2}}{2}\right)_{x}=f\left(x, u_{s}\right)-c_{f} u|u|
$$

where

$$
f=\frac{1}{a \sqrt{4 \pi \beta}} \exp \left[-\frac{\left(x-x_{s}+u_{s}^{-\alpha}\right)^{2}}{4 \beta}\right]
$$

represents the energy released by chemical reactions, with $x_{s}$ being the front position, $u_{s}$ the post-shock state, $\alpha$ and $\beta$ are parameters mimicking the role of activation energy and heat release, respectively, and $a=4(1+$

\footnotetext{
${ }^{1}$ In $[13$ some links were suggested between the shock-induced ignition problem in the context of Fickett's analog [14 and DDT. Here instead, we study the transition between the two steady solutions using a variation of the Rosales-Majda model [15].
} 
$\left.\operatorname{erf}\left(u_{s}^{-\alpha} / 2 \sqrt{\beta}\right)\right)$ a normalization constant so that $\int_{-\infty}^{\infty} f\left(x, u_{s}\right) d x=$ constant for any value of $u_{s}$. The values of $u_{s}$ and $\dot{x}_{s}$ are related through the RankineHugoniot jump conditions; asssuming a quiescent upstream state $(u=0)$, $\dot{x}_{s}=u_{s} / 2$. The variable $u$ can be thought of to be analogous to pressure in the real physical system, and $c_{f}$ is a parameter describing the strength of losses due to friction. Here we show that this simple model is also capable of qualitatively reproducing several of the distinguishing features observed in the DDT shown in Fig. 2 and 3 .

\section{Results and discussion}

\subsection{Existence of two traveling wave solutions}

An interesting mathematical consequence of the inclusion of sink terms such as $c_{f} u|u|$ in Eq. (1) (or in the reactive Euler equations, see [16 18]) is the existence of a new class of traveling wave solutions which propagate at speeds significantly lower than the CJ speed, and that exist in parallel with the classical ZND solution. The former waves, though unconditionally unstable, appear to play an important role in the DDT process, as can be seen by the presence of a rather stable phase in Fig. 3-Formation of waves ahead of the flame-- An example of the two types of traveling wave solutions

admitted by Eq. (1) (for $\alpha=1, \beta=0.1$ and $c_{f}=0.1$ ) is shown in Fig. 6 along with the spatial distributions of the contributions of each of the terms in Eq. (1), $u_{\text {terms }}$, to the structure of the wave. For more details on the numerical methodology used, steady and quasi-steady wave solutions, their linear stability and their dependence on $c_{f}$ readers are referred to [10].

There are notable differences in the structure of both traveling waves. 

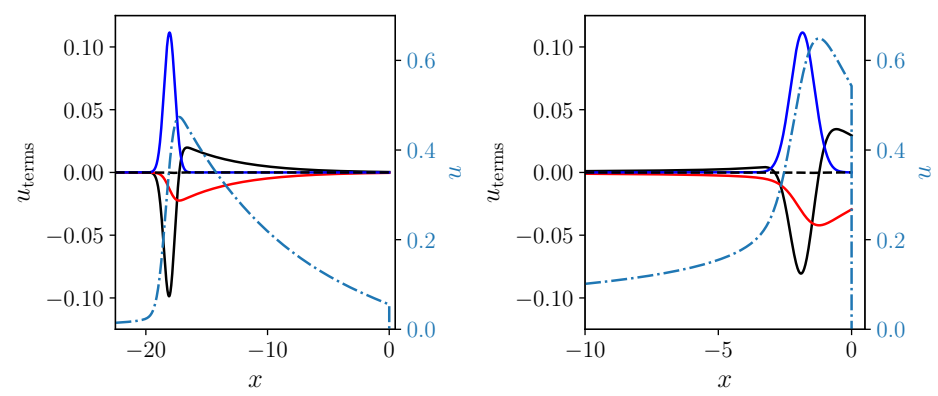

Figure 6: Structure of co-existing traveling wave solutions admitted by Eq. (1) - scalar variable, $u$ (dashed-dotted line), unsteady term, $u_{t}$ (dashed line), and budgets (solid lines): convection - black; friction losses - red; heat release - blue.

Specifically regarding the strengths of their leading shocks, reaction zone locations and the peaks attained in the scalar variable $u$. The fast wave exhibits the typical structure a detonation where chemistry is activated immediately behind the shock (i.e. peak in heat release located at $x=-1.86$ ), whereas in the slow wave the reaction zone trails far behind the leading shock at $x=-18.08$. The balancing terms up to the onset of the chemistry for both waves are the convection and friction losses; however, since for the slow wave the leading shock is not strong enough to initiate chemistry immediately behind it, the combined effect of friction and convection result in a long gradual increase in $u$ which finally culminates with heat release.

\subsection{Transition}

As mentioned above, qualitatively, the DDT process can be divided in three different phases. First, there is a slow regime composed of a typical flame which propagates with a speed on the order of tens of meters per second. The front propagation in this phase is driven primarily by transport phenomena (i.e. heat and mass), and is not captured by the averaged models 
composed of inviscid equations with sinks. The flame front then accelerates, generating a much faster front which propagates at nearly sonic speeds. At this point, it may overtake the pressure waves that the front itself generates, leading to the strengthening of the leading shock and eventually to the formation of a detonation wave.
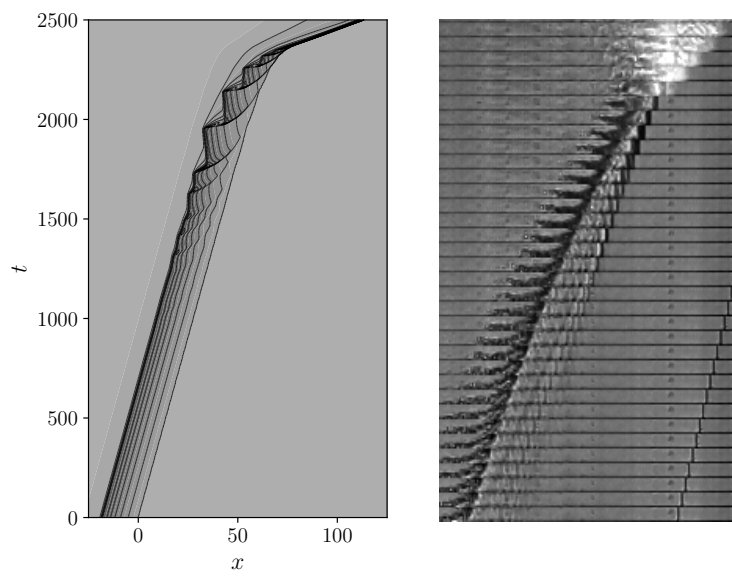

Figure 7: Qualitative comparison of the transition dynamics - Left: scalar model with generic losses. Right: experimental observations (right).

To investigate the transition we computed the time evolution of Eq. (1) using the slow traveling wave solution as initial conditions. Figure 7 shows the results of the model together with a side-by-side comparison of the section of experiments where the model is expected to be valid. We note that the numerical results show a very similar transition to that observed in the experiments. Initially weak waves emanating from the reaction zone towards the leading shock ahead, progressively become stronger gradually bringing the reaction zone closer to the leading shock until it finally transitions to the fast wave solution. Given the simplicity of the scalar equation used, it is quite remarkable that the dynamics of the later stages of the transition are 
well captured. The tractability of the model above allows to investigate the mechanisms of the transition in more detail.
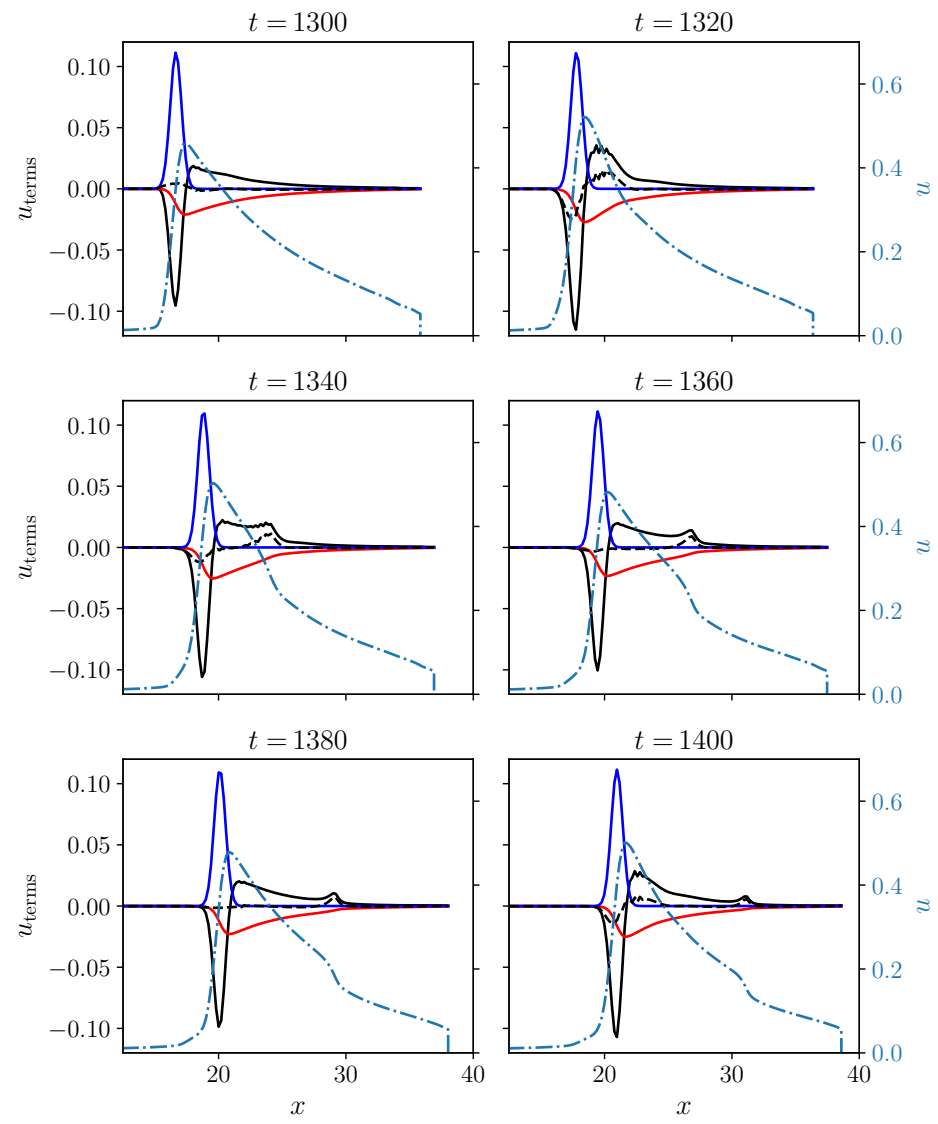

Figure 8: Wave structure at early stages - scalar variable, $u$ (dashed-dotted line), unsteady term, $u_{t}$ (dashed line) and budgets (solid lines): convection - black; friction losses - red; heat release - blue.

Figure 8 shows the initial development of unsteadiness within the reaction zone $\left(u_{t}>0\right)$ which subsequently results in a pulse in the scalar variable $u$ that travels toward the leading shock $(1300<t<1400)$. This imbalance brings about a positive/cyclic feedback that generates additional unsteadiness in the reaction zone whose end result is the birth of new pulses (see 
$t=1400$ at $x \sim 20)$.
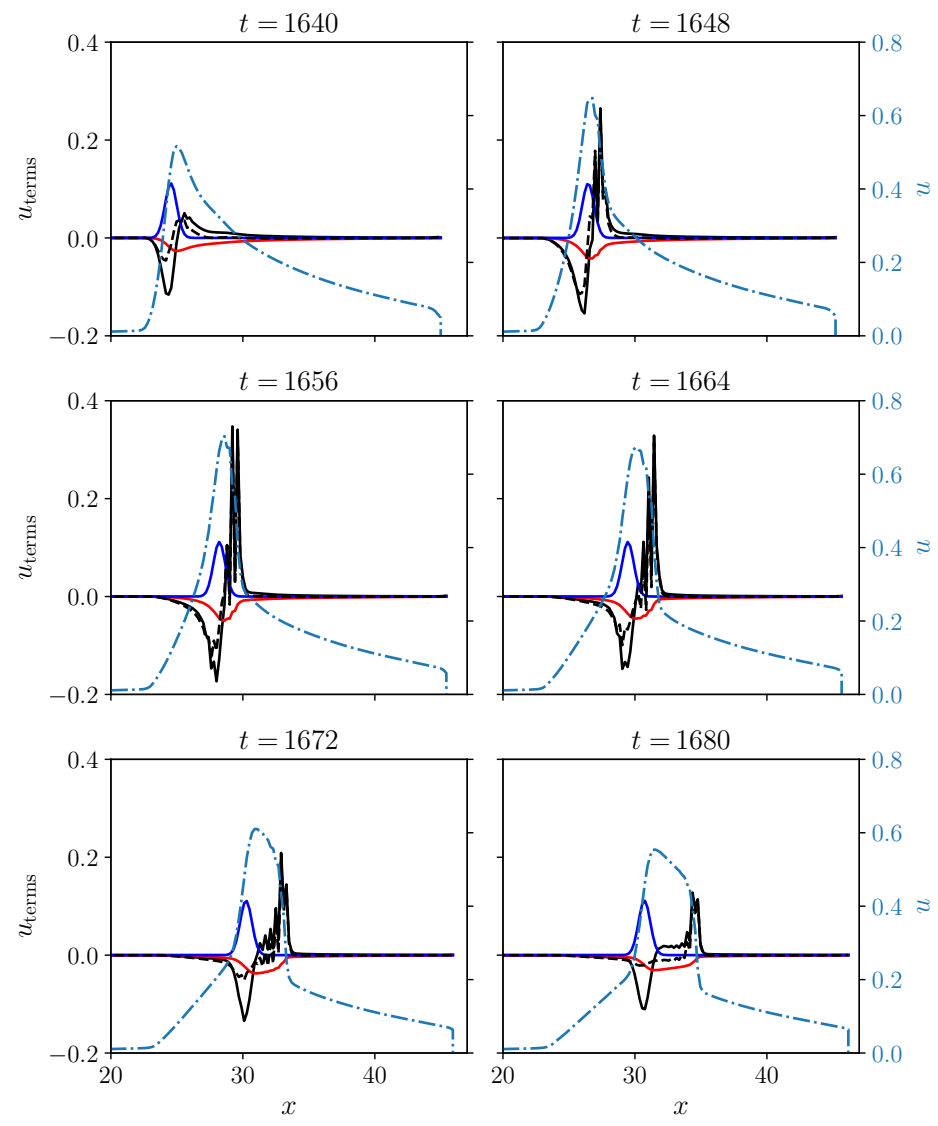

Figure 9: Wave structure at intermediate stages - scalar variable, $u$ (dashed-dotted line), unsteady term, $u_{t}$ (dashed line) and budgets (solid lines): convection - black; friction losses - red; heat release - blue.

As time evolves unsteadiness continues to increase generating ever stronger pulses (note the change in vertical scale in Fig. 9); the friction losses also increase as a result of the higher values of $u$ attained. The initial pulse-like perturbations that traveled toward the leading shock have now turned into shocks, in good qualitative agreement with the experimental observations (Fig. 7). 

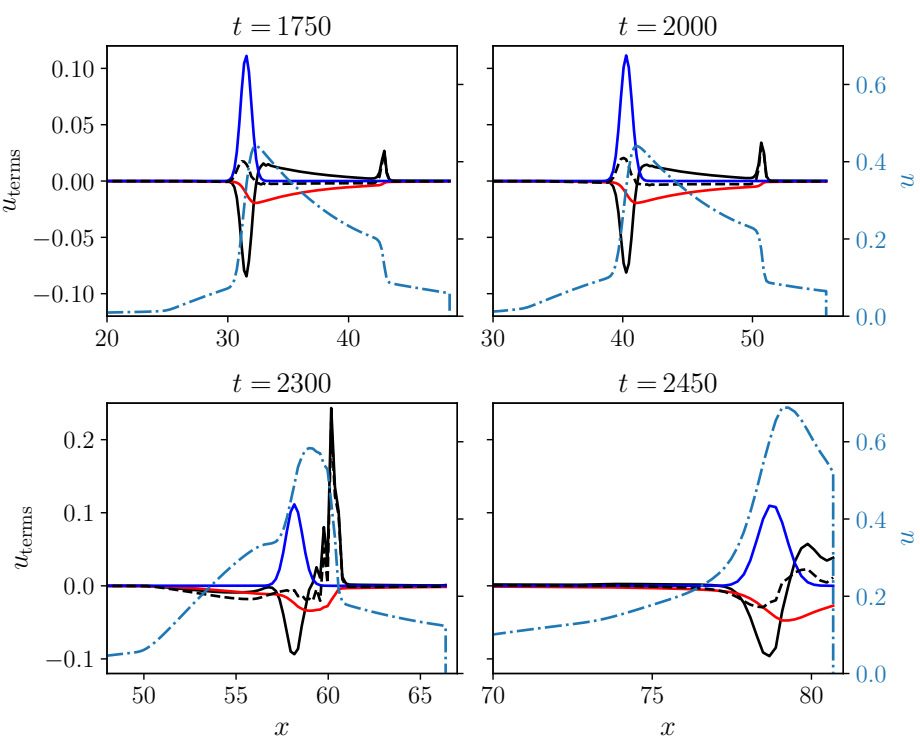

Figure 10: Wave structure during transition - scalar variable, $u$ (dashed-dotted line), unsteady term, $u_{t}$ (dashed line) and budgets (solid lines): convection - black; friction losses - red; heat release - blue.

In Fig. 10 the late stages of the transition are shown. Unsteadiness is always present within the reaction zone with a continuous feed of intermediate shocks toward the initial leading shock. The reaction zone progressively approaches the leading shock $(2000<t<2300)$, finally converging to the fast wave solution at $t=2450$. After 50 times units, $t=2500$, unsteadiness in the reaction zone disappears and the structure becomes that shown in Fig. 6.

The budgets above suggest that due to the unstable nature of the structure observed before transition (the presence of losses brings about an increased instability of the wave), once this state is engaged, any disturbance that results in fluctuations in heat release caused by perturbations in the reaction zone could lead to DDT. These fluctuations can physically arise from 
flow-, boundary layer-, transverse waves-flame interactions, instrinsic flame instabilities, as well as shock-boundary layer interactions. In the numerical simulations however, such instabilities are triggered by numerical discretization errors.

\section{Conclusions}

Interesting similarities between experimental observations of DDT in smooth narrow channels, and particular solutions of a simple one-dimensional scalar model have been shown. Our findings suggest that the transition between a fast, nearly sonic flame front, and a detonation wave may proceed through a sequence of intermittent pulses which are generated at the reaction zone and propagate towards the leading shock. Upon reaching the front, these waves appear to reduce the induction time, thus helping shorten the gap between the wave front and the reaction zone. After several cycles of the aforementioned dynamics, the distance between the reaction zone and the leading front becomes of the order of the reaction length, and a detonation wave is

initiated. Extension of this model to qualitatively capture the early stages of the process, where diffusive effects dominate, could be an interesting avenue worth exploring.

\section{Acknowledgments}

J. Melguizo-Gavilanes is grateful to M. Kuznetsov and J. Grüne for their support during his visit to KIT in Summer 2011. 


\section{References}

[1] R. Zipf Jr, V. Gamezo, M. Sapko, W. Marchewka, K. Mohamed, E. Oran, D. Kessler, E. Weiss, J. Addis, F. Karnack, et al., Methane-air detonation experiments at niosh lake lynn laboratory, Journal of Loss Prevention in the Process Industries 26 (2013) 295-301.

[2] G. Ciccarelli, S. Dorofeev, Flame acceleration and transition to detonation in ducts, Progress in Energy and Combustion Science 34 (2008) $499-550$.

[3] P. Wolański, Detonative propulsion, Proceedings of the Combustion Institute 34 (2013) 125-158.

[4] M. Liberman, Ma liberman, mf ivanov, ad kiverin, ms kuznetsov, aa chukalovsky, and tv rakhimova, deflagration-to-detonation transition in highly reactive combustible mixtures, acta astronaut. 67, 688 (2010)., Acta Astronaut. 67 (2010) 688.

[5] E. S. Oran, V. N. Gamezo, Flame acceleration and detonation transition in narrow tubes, in: Paper presented at 20th Int. Colloq. on the Dynamics of Explosions and Reactive Systems (ICDERS), McGill University, Montreal, 31 July - 5 August 2005.

[6] E. Dziemińska, A. K. Hayashi, Auto-ignition and ddt driven by shock wave-boundary layer interaction in oxyhydrogen mixture, International Journal of Hydrogen Energy 38 (2013) 4185-4193.

[7] T. Machida, M. Asahara, A. K. Hayashi, N. Tsuboi, Three-dimensional simulation of deflagration-to-detonation transition with a detailed chem- 
ical reaction model, Combustion Science and Technology 186 (2014) $1758-1773$.

[8] R. W. Houim, A. Ozgen, E. S. Oran, The role of spontaneous waves in the deflagration-to-detonation transition in submillimetre channels, Combustion Theory and Modelling 20 (2016) 1068-1087.

[9] A. Y. Poludnenko, T. A. Gardiner, E. S. Oran, Spontaneous transition of turbulent flames to detonations in unconfined media, Physical Review Letters 107 (2011) 054501.

[10] L. M. Faria, A. R. Kasimov, Qualitative modeling of the dynamics of detonations with losses, Proceedings of the Combustion Institute 35 (2015).

[11] Y. B. Zel'dovich, B. Gel'Fand, Y. M. Kazhdan, S. Frolov, Detonation propagation in a rough tube taking account of deceleration and heat transfer, Combustion, Explosion, and Shock Waves 23 (1987) 342-349.

[12] L. M. Faria, Qualitative and Asymptotic Theory of Detonations, PhD Thesis, King Abdullah University of Science and Technology, 2014.

[13] J. Tang, M. Radulescu, Dynamics of shock induced ignition in ficketts model: Influence of $\chi$, Proceedings of the Combustion Institute 34 (2013) 2035-2041.

[14] W. Fickett, Detonation in miniature, American Journal of Physics 47 (1979) 1050-1059. 
[15] R. R. Rosales, A. Majda, Weakly nonlinear detonation waves, SIAM Journal on Applied Mathematics 43 (1983) 1086-1118.

[16] I. Brailovskya, G. Sivashinsky, Hydraulic resistance and multiplicity of detonation regimes, Combustion and flame 122 (2000) 130-138.

[17] R. Semenko, L. Faria, A. R. Kasimov, B. Ermolaev, Set-valued solutions for non-ideal detonation, Shock Waves 26 (2016) 141-160.

[18] A. Sow, R. E. Semenko, A. R. Kasimov, On a stabilization mechanism for low-velocity detonations, Journal of Fluid Mechanics 816 (2017) $539-553$. 